\title{
DFT-D4 counterparts of leading meta-GGA and hybrid density functionals for energetics and geometries
}

\author{
Asim Najibi and Lars Goerigk* \\ School of Chemistry, The University of Melbourne, Parkville, Australia; \\ Ph: $+61-(0) 3-83446784$ \\ E-mail: lars.goerigk@unimelb.edu.au
}

\begin{abstract}
Previously, we introduced DFT-D3(BJ) variants of the B97M-V, $\omega$ B97X-V and $\omega \mathrm{B} 97 \mathrm{M}-\mathrm{V}$ functionals and assessed them for the GMTKN55 database [Najibi and Goerigk, J Chem. Theory Comput. 2018, 14, 5725]. In this study, we present DFT-D4 damping parameters to build the DFT-D4 counterparts of these functionals and assess these in comparison. We extend our analysis beyond GMTKN55 and especially turn our attention to enzymatically catalysed and metal-organic reactions. We find that B97M-D4 is now the second-best performing meta-GGA functional for the GMTKN55 database and it can provide noticeably better organometallic reaction energies compared to B97M-D3(BJ). Moreover, the aforementioned DFT-D3(BJ) based functionals have not been thoroughly assessed for geometries and herein we close this gap by analysing geometries of noncovalently bound dimers and trimers, peptide conformers, water hexamers and transition-metal complexes. We find that several of the B97(M)based methods - particularly the DFT-D4 versions - surpass the accuracy of previously studied methods for peptide conformer, water hexamer, and transition-metal complex
\end{abstract}


geometries, making them safe-to-use, cost-efficient alternatives to the original methods.

The DFT-D4 variants can be easily used with ORCA4.1 and above.

\section{Introduction}

Kohn-Sham density functional theory $(\mathrm{KS}-\mathrm{DFT})^{1}$ has become one of the most important and widely-used tools in molecular quantum chemistry. ${ }^{[2}$ All methods based on KS-DFT are approximations to the unknown true density functional, here labeled as density functional approximations (DFAs). Hundreds of DFAs have been developed, giving somewhat of a

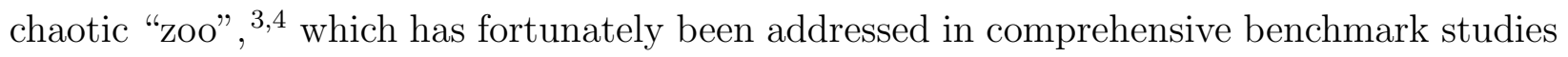
in order to identify robust DFAs for many types of chemical problems.

One of the main shortcomings of DFAs is their inability to properly describe Londondispersion interactions on their own. ${ }^{18}$ However, there are very reliable and widely used methods to correct this shortcoming. ${ }^{21 \mid 22}$ One very popular example is Grimme's DFTD3(BJ) ${ }^{23124}$ correction. It is based on the geometry of the system - taking into account the coordination number of each atom - uses dispersion coefficients derived from first principles, and requires the empirical fit of three DFA-specific parameters in the damping function, which connects the short-range component of the DFA with the long-range component of the dispersion correction; see Refs. 21 and 25 for reviews and more details. The extension of DFT-D3(BJ) is the relatively new DFT-D4, $26[27$ which has atomic-charge dependent dispersion coefficients and also requires the molecule's multiplicity as an input. By default it also uses a three-body term, ${ }^{23}$ which is only optional for DFT-D3(BJ).

Another very popular approach is the van-der-Waals-DFT (vdW-DFT) methodology, 28|29 where the semi-local exchange-correlation DFA is enhanced with a density-dependent nonlocalcorrelation component. This approach was popularised with the use of the VV10 nonlocal correlation kernel, 30 and subsequently generalised to many DFAs under the label "DFTNL". ${ }^{3132}$ DFT-NL methods can be very accurate with the right exchange-correlation func- 
tional, but come with a greater computational cost due to the evaluation of the nonlocal kernel; for comparisons between DFT-D3(BJ) and DFT-NL, see e.g. Refs. 31,32 and 33. The VV10 kernel is a two-body correction, but has been successfully enhanced with DFT-D3's three-body correction in benchmarking and applied studies. .34135

The VV10 kernel has gained increased attention through its use in the B97M-V, $\stackrel{[36}{\omega \mathrm{B} 97 \mathrm{X}-}$ $\mathrm{V}^{37}$ and $\omega \mathrm{B} 97 \mathrm{M}-\mathrm{V}^{38}$ DFAs developed by Mardirossian and Head-Gordon; for completeness reasons we also mention the $\omega \operatorname{Bg} 9 \mathrm{M}(2)$ double hybrid here, which however will not form part of our study! $!^{10}$ These functionals are based on the formalism of Becke's B97 ${ }^{39}$ DFA. B97M-V is a meta-generalised-gradient approximation (meta-GGA), as it incorporates the kinetic energy density. $\omega \mathrm{B} 97 \mathrm{X}-\mathrm{V}$ and $\omega \mathrm{B} 97 \mathrm{M}-\mathrm{V}$ are range-separated GGA and meta-GGA hybrid DFAs, respectively. Having been shown to be accurate DFAs by the developers,, 8 our group confirmed their high accuracy and robustness using the GMTKN $55^{3}$ database for general main-group chemistry, kinetics and noncovalent interactions (NCIs). 11 In that study, we additionally optimised the DFT-D3(BJ) correction to replace the nonlocal correlation component and showed that the resulting variants B97M-D3(BJ), $\omega$ B97X-D3(BJ) and $\omega$ B97M-D3(BJ) were viable and efficient alternatives to the original methods. We also found the DFT-D3(BJ) variants and the "-V" variants based on a post-self-consistent-field (post$\mathrm{SCF}$ ) nonlocal energy contribution can compute energies almost twice as fast as the originally published, full-SCF version. In a separate study by our group, the DFT-D3(BJ) variants were shown to be competitive or even better than the "-V" counterparts for enzymatically catalysed reactions. $\frac{40}{}$ Similarly, Iron and Janes benchmarked several density functionals for organometallic reaction energies (REs) and barrier heights (BHs), in which $\omega$ B97M-D3(BJ) was amongst the best methods for the REs and B97M-D3(BJ) and $\omega$ B97M-D3(BJ) were amongst the best for the BHs. $\underline{41}$

Having a good understanding of the applicability of the DFT-D3(BJ) variants of the B97(M) family of DFAs for (mostly) main-group energetics, we now intend to address their performance for geometries. Mardirossian and Head-Gordon provided a brief assessment of 
B97M-V and $\omega \mathrm{B} 97 \mathrm{X}-\mathrm{V}$ with the original full-SCF implementation for geometries, showing that they had the potential to be reliable methods. ${ }^{36137}$ Additionally, Kraus, Obenchain and Frank identified B97M-V as particularly useful for the geometries of noncovalently bound complexes. ${ }^{42}$ In 2013, Hujo and Grimme showed that the DFT-D3(BJ) and DFT-NL dispersion corrections provided very similar results for four functionals for the geometries of the S22 $2^{43}$ and S66 $6^{\sqrt[44]{4}}$ benchmark sets of noncovalently bound systems, the book, cage and prism water hexamers, and large transition-metal complexes. ${ }^{45}$ This makes it worthwhile to assess our DFT-D3(BJ) variants of the B97(M) type for geometries. If they provide reasonably accurate geometries they would offer a particularly cost-effective strategy compared to the original methods. As co-contributors to the quantum-chemical software ORCA, $\stackrel{46147}{w}$ we have also noticed an increased interest to use our DFT-D3(BJ) variants for geometries, ever since we included them in the program version 4.1 for energy calculations. In this study, we address if this can indeed be done safely to provide the users with a definite answer.

Given that we expect the new DFT-D4 to replace DFT-D3(BJ) in the future, we also present DFT-D4 variants of said DFAs, and we will particularly investigate if their atomiccharge dependence provide a potential improvement over the DFT-D3(BJ) variants for transition-metal systems.

In the following, we present the DFT-D4 damping parameters for the aforementioned DFAs. Next, we proceed with an assessment of the accuracy of all of the "-V", DFTD3(BJ) and DFT-D4 variants for energetics using the GMTKN55 database, ${ }^{3}$ a benchmark set containing enzymatically catalysed reactions,,$\underline{40148}$ and the MOR41 benchmark set of first-row metal-organic reactions. ${ }^{49}$ We then investigate their applicability for geometry optimisations using various benchmark sets where NCIs are significant, namely NCDT16 (noncovalently bound dimers and trimers) ${ }^{50}$ P26 (peptide conformers), ${ }^{5152}$ the book, cage and prism water hexamer isomers, $\frac{53}{5}$ and a set of first-row transition-metal complexes,,$\sqrt[54]{ }$ denoted as TMC32. .55 We are particularly interested in comparing the accuracy of the DFT-D3(BJ) and DFT-D4 DFAs for energetics and geometries, especially for the benchmark sets containing transition 
metals, due to the added charge- and total-spin dependence of DFT-D4.

\section{Technical details}

We tested twelve different methods that vary in either their underlying exchange-correlation expressions or the type of dispersion correction. They are PBE-D3(BJ), ${ }^{24 \mid 56}$ B3LYP-D3(BJ), 24/57/58

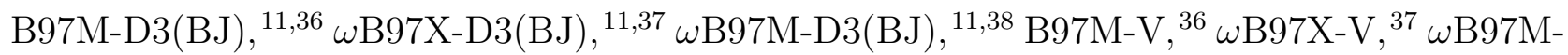
$\mathrm{V}, \stackrel{38}{38}$ DFT-D4 ${ }^{26 \mid 27}$ counterparts of all the B97(M)-based DFT-D3(BJ) ${ }^{23 \mid 24}$ methods, and $\omega \mathrm{B} 97 \mathrm{X}-\mathrm{D} 3, \sqrt[59]{ }$ which has been parameterised in the presence of the DFT-D3 zero-damping variant $^{23}$ and is therefore different from $\omega$ B97X-D3(BJ) and $\omega$ B97X-V. To avoid confusion, we denote $\omega$ B97X-D3 as $\omega$ B97X-D3(0) in the following. We decided to include PBE-D3(BJ), B3LYP-D3(BJ) and $\omega$ B97X-D3(0) in our analysis in order to assess whether our technical settings were compatible with previous studies that had assessed those methods, and to additionally compare our DFAs of interest to other popular functionals. Where useful, we also include published data for other DFAs in our analysis.

We determined the DFT-D4 damping parameters for B97M-D4, $\omega$ B97X-D4 and $\omega$ B97MD4 in a least-squares fit using the $\mathrm{S} 22 \times 5,60$ S66 $8^{44}$ and NCIBLIND ${ }^{61}$ NCI benchmark sets. These sets form the standard training set for both the latest DFT-D3 ${ }^{3 / 9111}$ and DFTD4 parametrisations. ${ }^{26127}$ The total energies for the exchange-correlation components were based on our previous work on the DFT-D3(BJ) parametrised variants $\frac{11}{11}$ and we combined those with DFT-D4 dispersion energies using Grimme's standalone program DFTD4. ${ }^{62}$ The exchange-correlation energies had been obtained with the def2-QZVP ${ }^{63}$ atomic-orbital (AO)

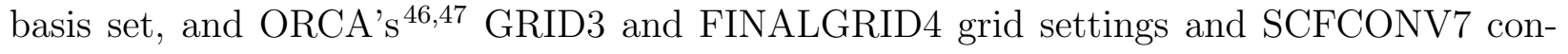
vergence criteria. ${ }^{11}$ Note that we used the default 'EEQ ${ }^{\sqrt{64}}$ version of DFT-D4 throughout this work.

In order to assess the methods for energetics, we used the GMTKN55 database,, 3 a benchmark set with models for enzymatically catalysed reactions, $\stackrel{40148}{ }$ and the MOR41 benchmark 
set for organometallic reactions. ${ }^{499}$ Again, the total energies for the underlying $(\omega)$ B97X/M single point calculations were taken from our previous works on GMTKN55 511 and the enzyme benchmark set ${ }^{40}$ and combined with new DFT-D4 dispersion energies. Calculations for MOR41 were calculated for this work with ORCA 4.2.1. $\stackrel{46147}{ }$ Results for GMTKN55 are based on the (aug'-)def2-QZVP ${ }^{6365}$ AO basis set, while the numbers for the enzyme benchmark set are based on def2-QZVP and those for MOR41 on def2-QZVPP. ${ }^{63}$ All numbers are based on ORCA's GRID3 and FINALGRID4 grid settings, standard grid settings for the VV10 nonlocal kernel, and SCFCONV7 convergence criteria.

The geometry optimisations were performed with the def2-TZVPD ${ }^{\sqrt{66}}$ basis set for NCDT16 and TMC32, $\frac{[54}{5}$ and with cc-pVTZ $\frac{67}{67}$ for P26 ${ }^{5152}$ and the water hexamers. ${ }^{[53}$ All DFT-D3/D4based calculations were done with ORCA 4.2.1 with the GRID7 and NOFINALGRID grid options, and VERYTIGHTOPT and SCFCONV7 convergence criteria. The optimisations for the "-V" variants were done with QCHEM ${ }^{[68}$ version 5.2 with the XC_GRID 3 grid setting and fully self-consistent standard VV10 nonlocal kernel grid (as intended by the developers). These settings closely matched the settings we used in ORCA for the energy calculations. Note that ORCA does not have an analytical gradient for DFT-NL methods yet, which is why we decided to include QCHEM results.

The AO basis sets were selected to make our studies comparable to previous studies which had used the benchmark sets that we have included. 3 [40149152155 The resolution-of-theidentiy (RI) approximation was used for the fitting of the DFT-D4 damping parameters, the single-point calculations and the geometry optimisations, with the corresponding auxiliary basis sets, unless specified. ${ }^{69}$ We utilised the RI approximation for Coulomb integrals (RI-J) for the GGA and meta-GGA functionals, and the range-separated hybrid functionals, and the chain-of-sphere extension ${ }^{[0]}$ with ORCA's default settings for exact-exchange integrals (RIJCOSX ) for the global hybrid B3LYP-D3(BJ). 


\section{Results and discussion}

\subsection{DFT-D4 parametrisation}

We begin our discussion by comparing the results of the fitting procedure of the DFT-D4 damping parameters for the B97(M) family of DFAs to the DFT-D3(BJ) data generated in our previous study ${ }^{11}$ In Table 1, we present the mean absolute deviations (MADs) of the DFT-D3(BJ) and DFT-D4 variants from reference values for the fit set and its individual subsets. The damping parameters and further statistics are shown in Tables S1-S5 in the Supporting Information (SI). We see that the DFT-D3(BJ) and DFT-D4 parameters fit very well, based on the MADs presented here and other statistics shown in the SI. Thus, we are confident in using our optimised DFT-D4 damping parameters. The reason that there is little change between the two corrections in the mean deviations (MDs), MADs, and root-meansquare deviations (RMSDs) is mostly due to the fact that the fit set contains typical, lighter elements and does not involve any charged or open-shell species. For a discussion on when to expect differences between the two corrections, see the original DFT-D4 literature. ${ }^{26127}$ That being said, we observe a reduction in the error ranges for the DFT-D4 methods of 0.44 $\mathrm{kcal} / \mathrm{mol}$ for B97M-D4, $1.45 \mathrm{kcal} / \mathrm{mol}$ for $\omega \mathrm{B} 97 \mathrm{X}-\mathrm{D} 4$, and $0.35 \mathrm{kcal} / \mathrm{mol}$ for $\omega \mathrm{B} 97 \mathrm{M}-\mathrm{D} 4$.

Calculations of our herein presented DFT-D4 variants can be performed with ORCA version 4.1 and higher even if the DFT-D4 parameters determined herein may not be hardcoded. We include an example input file in the SI (Fig. S1).

Table 1: Mean absolute deviations (kcal/mol) for the DFT-D3(BJ) and DFT-D4 variants from reference values for the semi-local exchange-correlation components of B97M-V, $\omega \mathrm{B} 97 \mathrm{X}-\mathrm{V}$ and $\omega \mathrm{B} 97 \mathrm{M}-\mathrm{V}(\mathrm{kcal} / \mathrm{mol})$. The def2-QZVP AO basis set was used.

\begin{tabular}{lcccccccc}
\hline & \multicolumn{3}{c}{ all } & \multicolumn{2}{c}{ S22x5 } & \multicolumn{2}{c}{ S66x8 } & \multicolumn{2}{c}{ NCIBLIND } \\
\hline & $-\mathrm{D} 3(\mathrm{BJ})^{a}$ & $-\mathrm{D} 4$ & -D3(BJ) $^{a}$ & $-\mathrm{D} 4$ & $-\mathrm{D} 3(\mathrm{BJ})^{a}$ & $-\mathrm{D} 4$ & $-\mathrm{D} 3(\mathrm{BJ})^{a}$ & $-\mathrm{D} 4$ \\
B97M & 0.13 & 0.16 & 0.15 & 0.16 & 0.12 & 0.16 & 0.16 & 0.15 \\
$\omega \mathrm{B} 97 \mathrm{X}$ & 0.15 & 0.17 & 0.18 & 0.18 & 0.14 & 0.17 & 0.19 & 0.15 \\
$\omega \mathrm{B} 97 \mathrm{M}$ & 0.16 & 0.12 & 0.16 & 0.12 & 0.15 & 0.12 & 0.18 & 0.15 \\
\hline
\end{tabular}

\footnotetext{
${ }^{a}$ Taken from ref. 11
} 


\section{$3.2 \quad$ Energetics}

\subsubsection{The GMTKN55 database}

We start the cross validation of our DFT-D4 variants with the help of the GMTKN55 database, which gives us a comprehensive overview of the methods' performance for general main-group chemistry, kinetics, and noncovalent interactions. GMTKN55 consists of 55 individual benchmark sets divided into five subcategories, namely basic properties and reactions of small systems, isomerisations and reactions of large systems, BHs, intermolecular and intramolecular NCIs. A total of 2462 single point calculations need to be carried out to assess a total of 1505 relative energies. The GMTKN55 database relies mostly on high-level wave-function theory reference values with only some experimental data points. In a series of four papers, $\sqrt[3419111]{ }$ our group - with the main study ${ }^{3}$ being a joint one with the Grimme group - have analysed 328 dispersion-corrected and uncorrected DFAs across all five rungs of Jacob's Ladder $; 71$ since then, others have also used the database for the evaluation and training of new methods, see e.g. Refs. 16 and 72 for select examples. Our previous studies excluded DFT-D4, but Grimme and co-workers established that several DFAs across Jacob's Ladder slightly improved with the DFT-D4 correction compared to the DFT-D3 correction for GMTKN55; ${ }^{27}$ this finding was more pronounced for the NCI benchmark sets. Focussing on the meta-GGA and hybrid-DFA rungs, we established the top-three DFT-D3 or VV10corrected DFAs for the entire database and its subcategories, with most of them being the original "V" corrected and our DFT-D3(BJ) version of the B97(M)-type DFAs. $\frac{11}{11}$ Herein, we test if any of our recommendations might change upon inclusion of DFT-D4 and whether the DFT-D4 variants outperform the DFT-D3(BJ) ones.

Looking at individual benchmark sets (see Tables S8-S13 in the SI for all data), we see that B97M-D4 and $\omega$ B97X-D4 perform significantly better compared to B97M-D3(BJ) and $\omega \mathrm{B} 97 \mathrm{X}-\mathrm{D} 3(\mathrm{BJ})$, respectively, for AL2X6 (dimerisation energies of AlX3 compounds) and ALK8 (reactions of alkaline compounds). Both benchmark sets are part of the "basic 
properties and reaction energies of small systems" category. The MADs of B97M-D3(BJ) for the AL2X6 and ALK8 benchmark sets are $5.45 \mathrm{kcal} / \mathrm{mol}$ and $9.85 \mathrm{kcal} / \mathrm{mol}$, whereas those of B97M-D4 are $1.63 \mathrm{kcal} / \mathrm{mol}$ and $2.70 \mathrm{kcal} / \mathrm{mol}$, competing with B97M-V with MADs of $1.56 \mathrm{kcal} / \mathrm{mol}$ and $2.71 \mathrm{kcal} / \mathrm{mol}$. Similarly, the MADs for $\omega$ B97X-D3(BJ) are $4.05 \mathrm{kcal} / \mathrm{mol}$ and $8.08 \mathrm{kcal} / \mathrm{mol}$, whereas those of $\omega \mathrm{B} 97 \mathrm{X}-\mathrm{D} 4$ are $2.80 \mathrm{kcal} / \mathrm{mol}$ and $3.01 \mathrm{kcal} / \mathrm{mol}$, thus much closer to those of $\omega \mathrm{B} 97 \mathrm{X}-\mathrm{V}$ with $1.21 \mathrm{kcal} / \mathrm{mol}$ and $0.95 \mathrm{kcal} / \mathrm{mol}$. Another example is the HEAVYSB11 set (dissociation energies in heavy-element compounds), which is also part of the "basic properties and reactions of small systems" category. Here, the MAD is $4.92 \mathrm{kcal} / \mathrm{mol}$ for B97M-D3(BJ), $2.57 \mathrm{kcal} / \mathrm{mol}$ for B97M-D4 and $2.20 \mathrm{kcal} / \mathrm{mol}$ for B97MV. Thus, we see several instances of improvement going from the DFT-D3(BJ) to their corresponding DFT-D4 methods for general main group chemistry. At the same time, we also find test sets, for which the opposite is true. For instance, for the "mindless" benchmark set MB16-43, B97M-D4 and B97M-V are with MADs of 37.54 and $35.40 \mathrm{kcal} / \mathrm{mol}$ worse than B97M-D3(BJ), with an MAD of $17.09 \mathrm{kcal} / \mathrm{mol}$. Also for MB16-43, $\omega$ B97M-D4 (MAD = $24.92 \mathrm{kcal} / \mathrm{mol})$ is worse than $\omega \mathrm{B} 97 \mathrm{M}-\mathrm{D} 3(\mathrm{BJ})(\mathrm{MAD}=21.65 \mathrm{kcal} / \mathrm{mol})$ and significantly worse than $\omega \mathrm{B} 97 \mathrm{M}-\mathrm{V}(\mathrm{MAD}=14.82 \mathrm{kcal} / \mathrm{mol})$. To put the magnitude of those values in a broader context, note that REs and deviations are generally larger for MB16-43 with only a few DFAs having MADs below $10 \mathrm{kcal} / \mathrm{mol}$, as discussed elsewhere. ${ }^{139}$ We refer to Tables S11-S13 for a detailed breakdown into how the DFT-D4 variants perform relatively to the other two variants for each individual benchmark set.

Above, we only discussed a few, select examples while Figure 1 represents a more compact analysis of potential improvements of the DFT-D4 variants over the other two. The figure shows the number of times the B97M-D4, $\omega$ B97X-D4 and $\omega$ B97M-D4 methods outperform their DFT-D3(BJ) and "-V" counterparts, based on the MAD of each of the 55 benchmark sets. In Tables S11-S13 we observe nearly identical trends from comparing RMSDs or error ranges with one another, which attests to the robustness of our MAD-based analysis in Figure 1. According to that figure, B97M-D4 clearly outperforms its DFT-D3 counterpart 


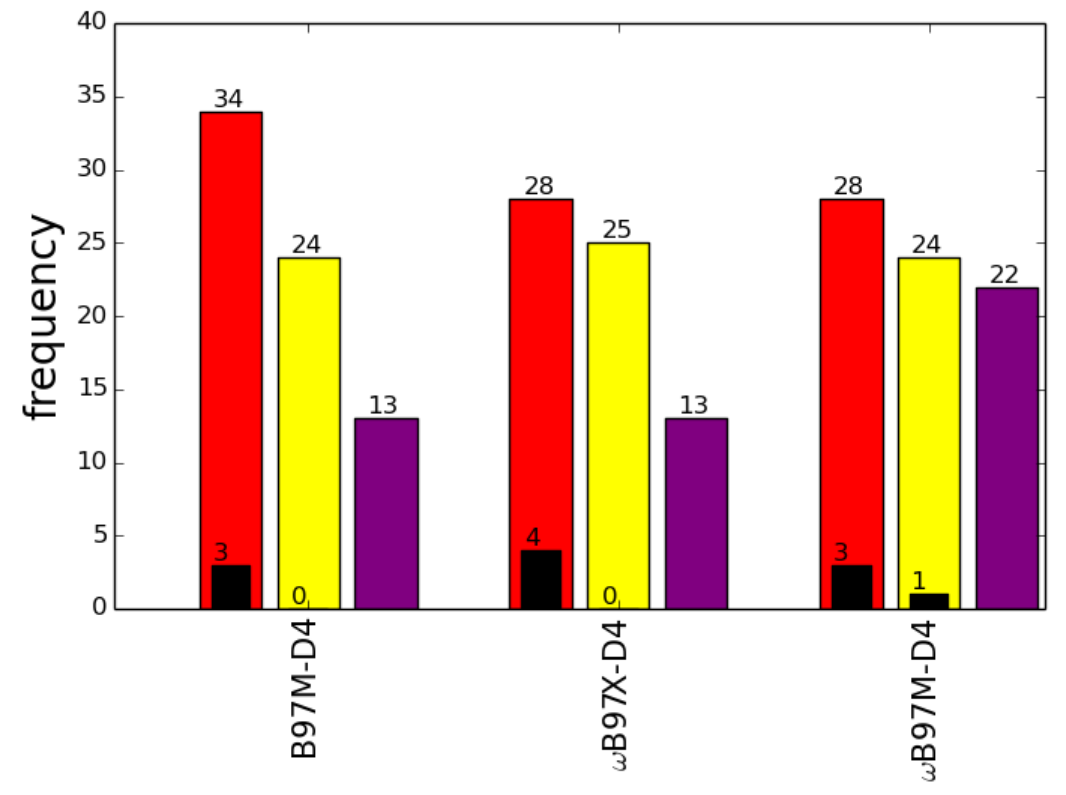

Figure 1: Frequency of a DFT-D4 variant outperforming its DFT-D3(BJ) (red) and "-V" (yellow) counterparts, based on MADs of individual benchmark sets in the GMTKN55 database. The purple bars show the number of cases in which a DFT-D4 method performs worse than its DFT-D3(BJ) and "-V" counterparts combined. The narrow black bars show how many times the DFT-D4 variant had equal MADs with either of its two counterparts. MADs for the DFT-D3 and "-V" versions were taken from Ref. 11

in 34 and the original B97M-V in 24 cases. Similar trends can also be seen for the other two DFAs (see Figure 1). Compared to both B97M-D3(BJ) and B97M-V, B97M-D4 is worse than both approaches in only 13 cases, however, note that compared to other published DFAs, its results still belong to some of the best reported. We observe the same for $\omega$ B97XD4 and note that the DFT-D4 counterpart of $\omega$ B97M seems to be the most negatively affected by inclusion of DFT-D4 with it being 22 times worse than both $\omega$ B97M-D3(BJ) and $\omega \mathrm{B} 97 \mathrm{M}-\mathrm{V}$. Table S6 lists an updated overview of the best meta-GGAs and hybrids for each of the 55 sets and thus replaces a similar table in the SI of Ref. 11. According to Table S6, B97M-D4 is now the best of all tested meta-GGAs for the sets dubbed ACONF (alkane conformers), ALK8, AMINO20x4 (amino-acid conformers), BH76RC (REs of various unimolecular, transfer, and substitution reactions), BHPERI (BHs of pericyclic reactions), BSR36 (bond-separation reactions), BUT14DIOL (butane-1,4,-diol conformers), and HAL59 
Table 2: WTMAD-2 values for the GMTKN55 database and its subcategories (kcal/mol). The (aug'-)def2-QZVP AO basis set was used.

\begin{tabular}{lccccccc}
\hline functional & basic & large & BHs & intramol. NCIs & intermol. NCIs & all NCIs & GMTKN55 \\
\hline PBE-D3(BJ) $^{a}$ & 6.51 & 12.36 & 18.36 & 10.21 & 9.58 & 9.90 & 10.32 \\
B3LYP-D3(BJ) $^{a}$ & 4.36 & 10.28 & 9.04 & 5.56 & 5.68 & 5.62 & 6.42 \\
B97M-D3(BJ) $^{b}$ & 4.54 & 9.08 & 7.95 & 6.83 & 6.15 & 6.49 & 6.49 \\
B97M-D4 $^{4}$ & 4.05 & 9.72 & 7.35 & 4.29 & 5.42 & 4.84 & 5.71 \\
B97M-V $^{b}$ & 3.68 & 9.31 & 7.53 & 3.56 & 5.74 & 4.63 & 5.46 \\
$\omega_{\text {B97X-D3(0) }}^{a}$ & 3.32 & 7.85 & 4.67 & 4.54 & 4.86 & 4.70 & 4.77 \\
$\omega_{\text {B97X-D3(BJ) }}$ & 3.47 & 5.64 & 3.68 & 4.96 & 4.53 & 4.75 & 4.35 \\
$\omega_{\text {B97X-D4 }}$ & 3.27 & 5.13 & 3.61 & 5.98 & 4.22 & 5.12 & 4.34 \\
$\omega_{\text {B97X-V }}^{a}$ & 3.34 & 6.68 & 4.21 & 3.03 & 3.63 & 3.32 & 3.98 \\
$\omega_{\text {B97M-D3(BJ) }}^{b}$ & 2.79 & 5.87 & 3.15 & 4.30 & 4.72 & 4.50 & 4.01 \\
$\omega_{\text {B97M-D4 }}$ & 2.81 & 5.83 & 3.11 & 5.43 & 3.99 & 4.72 & 4.09 \\
$\omega_{\text {B97M-V }}^{b}$ & 2.73 & 4.79 & 3.40 & 2.90 & 4.53 & 3.70 & 3.53 \\
\hline
\end{tabular}

${ }^{a}$ Taken from ref. [3. ${ }^{b}$ Taken from ref. [11.

(halogen-bonded dimers). In fact, B97M-D4 is the best of all tested DFAs across all five rungs of Jacob's Ladder for BSR36. wB97X-D4 is the best of all tested hybrid DFAs for BSR36, while $\omega$ B97M-D4 is the best of all tested DFAs across all rungs of Jacob's Ladder for ADIM6 (alkane dimers).

As outlined in Ref. 3, it is in principle more useful to combine all individual MADs to one final number. This is done in Table 2, which shows the weighted total mean absolute deviations (WTMADs) for the assessed methods for GMTKN55 and its subcategories. WTMADs allow to represent the individual statistics for all benchmark sets with one final number either for the entire database or one of its subcategories. We use the WTMAD-2 scheme presented in the original GMTKN55 study - the weighting for each benchmark set in the GMTKN55 database depends on the number of REs and the average absolute reference REs. $\stackrel{3}{\text { PBE- }}$ D3(BJ) and B3LYP-D3(BJ) results are included to facilitate the comparison with popular methods and to repeat our message that popularity of a DFA does not justify that it should be applied. For instance, amongst the 328 DFAs tested by our group prior to this study, PBE-D3(BJ) and B3LYP-D3(BJ) ranked in 140th and 77th position, respectively.4

We see from Table 2 that B97M-D4, $\omega$ B97X-D4 and $\omega$ B97M-D4 perform similarly to 
their DFT-D3(BJ) and "-V" counterparts. We note that there are a few cases for which a DFT-D4 method surpasses the accuracy of the original approaches, whereas the corresponding DFT-D3(BJ) method trails the latter. For example, for the basic-properties subcategory, $\omega$ B97X has WTMAD-2 values of 3.47, 3.27 and $3.34 \mathrm{kcal} / \mathrm{mol}$ for -D3(BJ), -D4 and $-\mathrm{V}$, respectively. For the BHs, we obtain WTMAD-2 values of 7.95, 7.35 and 7.53 $\mathrm{kcal} / \mathrm{mol}$ for B97M-D3(BJ), B97M-D4 and B97M-V. Lastly, there are two types of underlying exchange-correlation approximations that are noteworthy for the treatment of intermolecular NCIs, namely B97M-D3(BJ), B97M-D4 and B97M-V with WTMAD-2 $=6.15,5.42$ and 5.74 $\mathrm{kcal} / \mathrm{mol}$, respectively, as well as $\omega \mathrm{B} 97 \mathrm{M}-\mathrm{D} 3(\mathrm{BJ}), \omega \mathrm{B} 97 \mathrm{M}-\mathrm{D} 4$ and $\omega \mathrm{B} 97 \mathrm{M}-\mathrm{V}$ with values of $4.72,3.99$ and $4.53 \mathrm{kcal} / \mathrm{mol}$.

Table S7 provides us with an updated list of the top-three meta-GGA and hybrid functionals for each of the categories of GMTKN55 and the entire database. B97M-D4 is now the third-best meta-GGA functional for basic properties and reaction energies of small systems, with a WTMAD-2 of $4.05 \mathrm{kcal} / \mathrm{mol}$. $\omega \mathrm{B} 97 \mathrm{X}-\mathrm{D} 4$ is now the third-best hybrid functional for REs of large systems and isomerization energies, with a WTMAD-2 of $5.13 \mathrm{kcal} / \mathrm{mol}$. B97M-D4 and $\omega$ B97M-D4 are particularly useful for BHs, surpassing all previously assessed meta-GGA and hybrid functionals, respectively, to be the best in their classes-B97M-D4 and $\omega$ B97M-D4 have WTMAD-2 values of 7.35 and $3.11 \mathrm{kcal} / \mathrm{mol}$, respectively. B97MD4 is the second-best meta-GGA functional for intramolecular NCIs, with WTMAD-2 = $4.29 \mathrm{kcal} / \mathrm{mol}$. B97M-D4 has become the highest ranked meta-GGA functional for intermolecular NCIs, with a WTMAD-2 of $5.42 \mathrm{kcal} / \mathrm{mol}$, surpassing B97M-D3(BJ) which has a WTMAD-2 of $5.74 \mathrm{kcal} / \mathrm{mol}$. $\omega \mathrm{B} 97 \mathrm{M}-\mathrm{D} 4$ is now the second-best DFA for intermolecular NCIs with a WTMAD-2 $=3.99 \mathrm{kcal} / \mathrm{mol}$, behind $\omega \mathrm{B} 97 \mathrm{X}-\mathrm{V}$ with a WTMAD-2 of 3.62 $\mathrm{kcal} / \mathrm{mol}$. Looking at all NCIs combined, B97M-D4 is the second-best meta-GGA functional, with a WTMAD-2 of $4.84 \mathrm{kcal} / \mathrm{mol}$, clearly surpassing B97M-D3(BJ), with a WTMAD-2 of $6.49 \mathrm{kcal} / \mathrm{mol}$. Lastly, we note the updated top-three meta-GGA functionals for the entire GMTKN55 database, which all belong to the same underlying exchange-correlation 
DFA. These are B97M-V, B97M-D4 and B97MD3(BJ), with WTMAD-2 values of 5.46, 5.71 and $6.49 \mathrm{kcal} / \mathrm{mol}$, respectively. Thus, our updates for GMTKN55 indicate the usefulness of DFT-D4 for B97M, $\omega$ B97X and $\omega$ B97M over DFT-D3(BJ) and in some cases "-V," in various areas of thermochemistry and NCIs, and especially for the calculation of BHs.

\subsubsection{Enzymatically catalysed reactions}

The benchmark set of enzymatically catalysed reactions consists of five chemical problems taken from the literature ${ }^{73}$ but provided with reoptimised structures and accurate DLPNO-CCSD $(\mathrm{T})^{79}$ reference energies at the complete-basis-set (CBS) limit in 2019. 40148 Each of the reactions is represented by different models of varying sizes to take into the account the effects of the amino-acid residues close to the active centres. The set consists of 16 BHs and 12 REs; 4 BHs and 2 REs belong to model systems containing two $\mathrm{Zn}^{2+}$ ions each in their active centres, the other models do not contain any transition-metal ions. The 2019 study considered a total of 35 DFAs including the VV10 and DFT-D3(BJ) corrected methods that our current study focuses on. B97M-D3(BJ) was found to be the best-performing meta-GGA functional, and $\omega$ B97M-V was found to be the second-best performing hybrid functional. Here, we extend this benchmark study to our DFT-D4 methods and the popular functionals which we have chosen to include. In addition to partitioning the set into BHs and REs, we also partition it into systems with and without the $\mathrm{Zn}^{2+}$-containing models. This shall serve as a preliminary assessment of the effect of the charge-dependency of the DFT-D4 dispersion correction, which presumably benefits transition-metal compounds. The MADs for the different partitions and for the entire benchmark set are shown in Table 3 . All statistical results are shown in Tables S11-S15.

Having a close look at the $\mathrm{Zn}^{2+} /$ no- $\mathrm{Zn}^{2+}$ partitioning, we see that the underlying exchangecorrelation DFA — but to an extent also the type of dispersion correction - determines if the MADs of both subsets are similar or very different. For instance, both MADs for B97MD4 and $\omega$ B97X-D4 are comparable with differences of 0.34 and $0.08 \mathrm{kcal} / \mathrm{mol}$, respectively 
Table 3: MADs for the benchmark set of enzymatically catalysed reactions $(\mathrm{kcal} / \mathrm{mol})$. The def2-QZVP AO basis set was used.

\begin{tabular}{|c|c|c|c|c|c|}
\hline functional & all & $\mathrm{BHs}$ & REs & $\mathrm{Zn}^{2+}$ only & without $\mathrm{Zn}^{2+}$ \\
\hline PBE-D3(BJ) ${ }^{a}$ & 5.33 & 6.35 & 3.97 & 3.49 & 5.84 \\
\hline B3LYP-D3(BJ) ${ }^{a}$ & 3.76 & 4.33 & 2.99 & 4.19 & 3.64 \\
\hline B97M-D3(BJ) ${ }^{a}$ & 2.87 & 2.95 & 2.78 & 2.06 & 3.10 \\
\hline B97M-D4 & 3.28 & 3.09 & 3.54 & 3.55 & 3.21 \\
\hline B97M-V ${ }^{a}$ & 2.97 & 3.02 & 2.90 & 2.77 & 3.02 \\
\hline$\omega \mathrm{B} 97 \mathrm{X}-\mathrm{D} 3(\mathrm{BJ})^{a}$ & 1.75 & 1.58 & 1.97 & 1.57 & 1.79 \\
\hline$\omega \mathrm{B} 97 \mathrm{X}-\mathrm{D} 4$ & 1.74 & 1.56 & 1.97 & 1.76 & 1.84 \\
\hline$\omega \mathrm{B} 97 \mathrm{X}-\mathrm{V}^{a}$ & 1.88 & 1.62 & 2.23 & 2.24 & 1.78 \\
\hline$\omega \mathrm{B} 97 \mathrm{M}-\mathrm{D} 3(\mathrm{BJ})^{a}$ & 1.35 & 1.16 & 1.60 & 2.79 & 0.96 \\
\hline$\omega B 97 M-D 4$ & 1.39 & 1.15 & 1.72 & 3.19 & 0.99 \\
\hline$\omega B 97 \mathrm{M}-\mathrm{V}^{a}$ & 1.34 & 1.25 & 1.46 & 2.15 & 1.12 \\
\hline$\omega \mathrm{B} 97 \mathrm{X}-\mathrm{D} 3(0)^{a}$ & 1.80 & 1.67 & 1.99 & 4.17 & 1.16 \\
\hline
\end{tabular}

aTaken from Ref. 40

(Table 3). Contrary to that, the MAD for the $\mathrm{Zn}^{2+}$-containing subset is much higher than that of main-group-based systems for $\omega$ B7M-D4 $(\mathrm{MAD}=3.19 \mathrm{kcal} / \mathrm{mol}$ vs. $\mathrm{MAD}=0.99$ $\mathrm{kcal} / \mathrm{mol}$ ). Marginal differences of up to $0.25 \mathrm{kcal} / \mathrm{mol}$ between the MADs for both subsets can also be found for B97M-V and $\omega$ B97X-D3(BJ), while differences of around $0.5 \mathrm{kcal} / \mathrm{mol}$ are observed for $\omega$ B97X-V. All other DFT-D3(BJ) and "-V" type DFAs have larger differ-

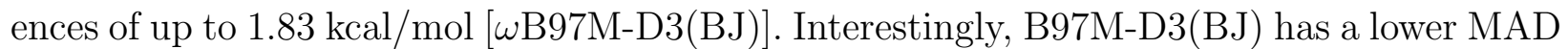
for the $\mathrm{Zn}^{2+}$-containing subset than for the remaining systems, which is the opposite trend compared to its DFT-D4 and "V" variants. We note that differences between both subsets are also sizeable for PBE-D3(BJ), B3LYP-D3(BJ), and $\omega$ B97X-D3(0). Our results come as a surprise, considering the DFT-D4 correction utilises atomic partial charges, which would have made us hope that the $\mathrm{Zn}^{2+}$ centres were described better. That being said, our sample size is most likely too small and it is worthwhile to consider a more extensive benchmark set for transition-metal complexes in order to test the functionals more thoroughly, which is done in the following section.

We conclude this section by comparing the DFT-D4-based results with the previously published recommendations for the total set. At the meta-GGA level, the initial recommen- 
dation to use B97M-D3(BJ) remains unchanged. $\omega \mathrm{B} 97 \mathrm{M}-\mathrm{V}$ is still the best hybrid DFA, closely followed by its DFT-D3(BJ) and DFT-D4 variants, which could safely be used too.

\subsubsection{The MOR41 set}

The MOR41 benchmark set of metal-organic reactions was presented by Grimme and coworkers alongside a benchmark study of 54 dispersion-corrected DFAs. ${ }^{49}$ The set contains 41 closed-shell organometallic reactions resembling common chemical transformations used in transition-metal chemistry and catalysis. The MOR41 set relies on DLPNO-CCSD $(\mathrm{T})^{[79}$ reference values at the CBS limit. Amongst the assessed methods, Grimme and co-workers also examined the PBE-D3(BJ), B3LYP-D3(BJ) and $\omega$ B97X-V DFAs, which we have included in our study. The authors showed that $\omega \mathrm{B} 97 \mathrm{X}-\mathrm{V}$ was found to be the best hybrid functional. Additionally, Iron and Janes benchmarked several density functional approximations including B97M-V, B97M-D3(BJ), $\omega$ B97X-V, $\omega$ B97X-D3(BJ), $\omega$ B97M-V and $\omega$ B97M-D3(BJ) for this set. ${ }^{41}$ Their analysis showed B97M-V to be amongst the best meta-GGAs, and $\omega$ B97X$\mathrm{V}, \omega \mathrm{B} 97 \mathrm{M}-\mathrm{V}$ and $\omega \mathrm{B} 97 \mathrm{M}-\mathrm{D} 3(\mathrm{BJ})$ to be amongst the best hybrid DFAs. It is therefore worthwhile to also assess our DFT-D4 variants for this set. Note that all statistical results presented herein are all from our own calculations (see Table S19 for all results), but comparable to the numbers published by Grimme and co-workers - for example, the RMSD of $\omega \mathrm{B} 97 \mathrm{X}-\mathrm{V}$ is $2.79 \mathrm{kcal} / \mathrm{mol}$ in both our study and that of Grimme and co-workers.

Our hypothesis is to see an improvement when replacing the DFT-D3(BJ) with the DFT-D4 correction for the MOR41 and the B97(M) family of DFAs due to the reasons stated earlier. The MADs depicted in Figure 2 indicate that this is indeed the case for the meta-GGA functionals, with B97M-D3(BJ) having an MAD of $5.52 \mathrm{kcal} / \mathrm{mol}$ and B97M-D4 an MAD of $3.76 \mathrm{kcal} / \mathrm{mol}$. By changing the type of dispersion correction B97M-D4 has become competitive with B97M-V $(\mathrm{MAD}=3.86 \mathrm{kcal} / \mathrm{mol})$. Contrary to those findings, we only see a slight improvement when going from $\omega$ B97X-D3(BJ) to $\omega$ B97X-D4, which still has an MAD that is $3.00 \mathrm{kcal} / \mathrm{mol}$ greater than that of $\omega \mathrm{B} 97 \mathrm{X}-\mathrm{V}$. $\omega \mathrm{B} 97 \mathrm{M}-\mathrm{D} 3(\mathrm{BJ})$ and $\omega \mathrm{B} 97 \mathrm{M}-$ 


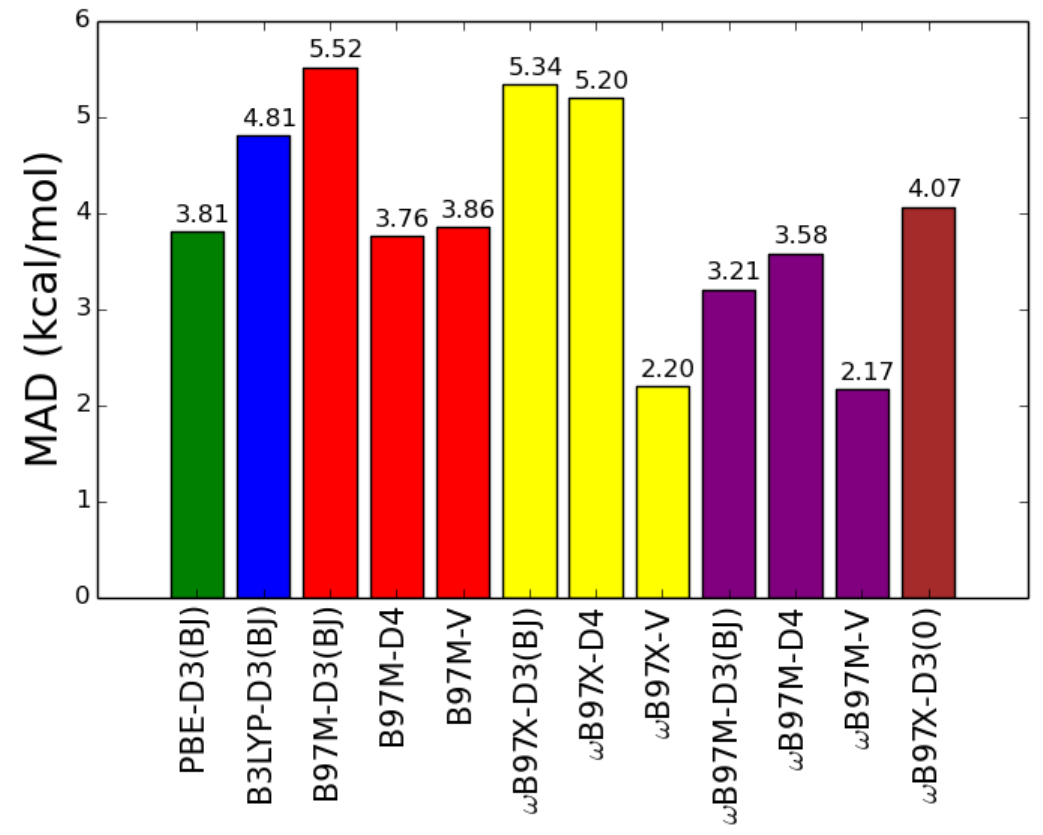

Figure 2: Mean absolute deviations $(\mathrm{kcal} / \mathrm{mol})$ for the various methods tested in this study for the MOR41 benchmark set. The def2-QZVPP AO basis set was used

D4 perform similarly for MOR41, with the MAD of $\omega$ B97M-D3(BJ) being better by 0.37 $\mathrm{kcal} / \mathrm{mol}$. Based on all the assessed methods, we recommend that the DFT-D4 methods be used as opposed to the DFT-D3(BJ) methods for systems reminiscent of those in MOR41, since there is either improvement or very little change. While we recommend all original "-V" methods for the MOR41 set, we can also safely recommend B97M-D4 as a cost-efficient alternative if hybrid DFAs are not applicable to a particular problem.

\subsection{Geometries}

\subsubsection{The NCDT16 set}

The NCDT16 benchmark set consists of 16 noncovalently bound dimers and trimers. In 2018, Kraus and Frank assessed 89 computational methods on this set, $\stackrel{42}{85}$ of which were DFAs. They found B97M-V to be the best method, with $\omega$ B97M-V and $\omega$ B97X-V being sixth and seventh best, respectively. In our study, we include the DFT-D3(BJ) and DFT-D4 
counterparts of these functionals using ORCA, and redo the VV10-type calculations using QCHEM. The analysis of the NCDT16 benchmark set involves calculating the MAD for each system based on designated interatomic distances - within each monomer and between the individual monomers - and then averaging these over the sixteen systems, giving an average MAD. Reference values are theoretically back-corrected experimental data. $\underline{50}$

Our results are shown in Table 4. We see that the DFT-D3(BJ) and DFT-D4 counterparts of B97M-V, $\omega \mathrm{B} 97 \mathrm{X}-\mathrm{V}$ and $\omega \mathrm{B} 97 \mathrm{M}-\mathrm{V}$ come close to the original methods, yet do not outperform them. For example, $\omega$ B97X-D4 has an average MAD that is only $0.001 \AA$ higher than that of $\omega \mathrm{B} 97 \mathrm{X}-\mathrm{V}$, whereas the value for $\omega \mathrm{B} 97 \mathrm{M}-\mathrm{D} 4$ is $0.046 \AA$ higher than for $\omega \mathrm{B} 97 \mathrm{M}-$ V. However, all of the DFT-D3(BJ) and DFT-D4 methods outperform $\omega$ B97X-D3(0), with the smallest difference being $0.044 \AA$ [between $\omega$ B97M-D4 and $\omega$ B97X-D3(0)]. This demonstrates that we may use the DFT-D3(BJ) and DFT-D4 variants for geometry optimisations of similar noncovalently bound systems with only a small loss of accuracy.

Table 4: Average MADs for the NCDT16 benchmark set $(\AA)$. The def2-TZVPD AO basis set was used.

\begin{tabular}{lc}
\hline functional & av. MAD \\
\hline PBE-D3(BJ) & 0.073 \\
B3LYP-D3(BJ) & $0.302^{a}$ \\
B97M-D3(BJ) & 0.053 \\
B97M-D4 & 0.037 \\
B97M-V & 0.032 \\
$\omega B 97 X-D 3(B J)$ & 0.057 \\
$\omega B 97 X-D 4$ & 0.050 \\
$\omega B 97 X-V$ & 0.049 \\
$\omega B 97 M-D 3(B J)$ & 0.080 \\
$\omega B 97 M-D 4$ & 0.086 \\
$\omega B 97 M-V$ & 0.040 \\
$\omega B 97 X-D 3(0)$ & 0.130 \\
\hline
\end{tabular}

${ }^{a}$ Without the RIJCOSX approximation the av. MAD is $0.043 \AA$.

While we do not see any error during our calculations, this may be worth investigating further. 


\subsubsection{The P26 set}

The P26 set consists of 26 conformers of five peptides with aromatic side chains. Initially proposed by Hobza and co-workers, ${ }^{51}$ Goerigk and Reimers reoptimised all structures at the MP2/aug-cc-pVTZ level to subsequently study the effect of London-dispersion corrections and the basis-set superposition error on these structures for a handful of DFAs for triple- $\zeta$ (TZ) and smaller AO basis sets. $\frac{52}{52}$ The reference level of theory was deemed to be reasonably reliable for the questions that Goerigk and Reimers wanted to answer. Moreover, the recommendations based on P26 could later be verified for larger protein fragments that allowed a direct comparison with experimental structures, $\frac{80}{20}$ wich gives further credibility to the usefulness of those reference structures. As our main purpose here is the comparison between the different dispersion-corrected methods, we are confident that the same reference structures are still useful. The analysis for P26 is carried out by calculating the RMSDs with respect to the reference structure for each conformer and to then form the average RMSD over the entire set.

The average RMSDs are shown in Table 5 and include values for PBE-D3(BJ), B3LYPD3(BJ), and PW6B95-D3(BJ) 2481 taken from Ref. 52. The latter was shown to be particularly reliable at the TZ level, not only for P26 but also other structures investigated as part of the same study ${ }^{\sqrt{52}}$ as well as for larger protein fragments. $\stackrel{80}{ }$ PW6B95-D3(BJ) has an average RMSD of $0.111 \AA$ and herein we present several methods that surpass this value,

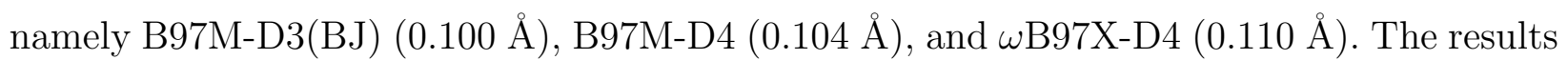
for the meta-GGA variants are particularly promising, as they offer a cheaper route towards obtaining reliable structures of larger polypeptides. This finding warrants further investigation along the lines of their potential for usage in X-ray quantum refinement as suggested in Ref. 80.

When comparing the different dispersion corrections with one another, we see that the DFT-D3(BJ) and DFT-D4 counterparts of B97M-V and $\omega$ B97X-V perform slightly better than their corresponding "V" methods. The average RMSD is $0.100 \AA$ and $0.104 \AA$ for 
Table 5: Average RMSDs for the P26 benchmark set $(\AA)$. The cc-pVTZ AO basis set was used.

\begin{tabular}{lc}
\hline functional & av. RMSD \\
\hline PBE-D3(BJ) $^{a}$ & 0.196 \\
B3LYP-D3(BJ) $^{a}$ & 0.120 \\
B97M-D3(BJ) & 0.100 \\
B97M-D4 & 0.104 \\
B97M-V & 0.145 \\
$\omega$ B97X-D3(BJ) & 0.140 \\
$\omega$ B97X-D4 & 0.110 \\
$\omega$ B97X-V & 0.148 \\
$\omega$ B97M-D3(BJ) & 0.123 \\
$\omega$ B97M-D4 & 0.134 \\
$\omega$ B97M-V & 0.125 \\
$\omega$ B97X-D3(0) & 0.160 \\
PW6B95-D3(BJ $)^{a}$ & 0.111 \\
\hline
\end{tabular}

${ }^{a}$ Taken from ref. 52

B97M-D3(BJ) and B97M-D4, respectively, whereas the average RMSD of B97M-V is 0.145 $\AA$. Similarly, the average RMSD is $0.140 \AA$ and $0.110 \AA$ for $\omega$ B97X-D3(BJ) and $\omega$ B97X$\mathrm{D} 4$, respectively, whereas the average RMSD of $\omega \mathrm{B} 97 \mathrm{X}-\mathrm{V}$ is $0.148 \AA$. In contrast to those findings, we also find that the DFT-D3(BJ) and DFT-D4 counterparts of $\omega$ B97M-V only perform similarly to $\omega \mathrm{B} 97 \mathrm{M}-\mathrm{V}$, with respective average RMSDs of $0.123 \AA, 0.134 \AA$ and $0.125 \AA$.

\subsubsection{Water hexamers}

Accurate experimental estimates of the oxygen-oxygen distances in three water-hexamer isomers (book, cage and prism) were provided via broadband rotational spectroscopy. 53 Those values were used as a benchmark in quantum-chemical optimisation studies using DFT-NL by Hujo and Grimme ${ }^{\sqrt{45}}$ as well as by Goerigk and Reimers in their study on dispersion and basis-set effects that also included the P26 set. ${ }^{52}$ Following the strategy of those previous papers, the average oxygen-oxygen distances are calculated for each isomer and then again averaged over the three systems. This is done for both the reference and 
Table 6: MDs and MADs $(\AA)$ for the water hexamers. For each hexamer the average deviation from average $\mathrm{O}-\mathrm{O}$ distances for each system are calculated. These individual average deviations are used to calculate the MDs and MADs. The cc-pVTZ AO basis set was used.

\begin{tabular}{lcc}
\hline functional & MD & MAD \\
\hline PBE-D3(BJ $^{a}$ & -0.05 & 0.05 \\
B3LYP-D3(BJ) $^{a}$ & -0.02 & 0.02 \\
B97M-D3(BJ) & 0.01 & 0.01 \\
B97M-D4 & 0.01 & 0.01 \\
B97M-V & 0.00 & 0.01 \\
$\omega$ B97X-D3(BJ) & 0.00 & 0.00 \\
$\omega$ B97X-D4 & 0.00 & 0.00 \\
$\omega$ B97X-V & -0.01 & 0.01 \\
$\omega$ B97M-D3(BJ) & -0.01 & 0.01 \\
$\omega$ B97M-D4 & -0.01 & 0.01 \\
$\omega$ B97M-V & -0.02 & 0.02 \\
$\omega$ B97X-D3(0) & -0.03 & 0.03 \\
PBE0-D3(BJ) & -0.04 & 0.04 \\
PW6B95-D3(BJ) & \\
BHLYP-D3(BJ) $^{a}$ & 0.01 & 0.01 \\
la $^{a}$ Iakn & -0.02 & 0.02 \\
\hline
\end{tabular}

${ }^{a}$ Taken from ref. 52

quantum-chemically optimised structures.

Table 6 shows the MDs over those three averaged values of the assessed DFT methods with respect to the reference values; note that a negative MD indicates an over-binding (too short distances) tendency. B97M-V outperforms its DFT-D3(BJ) and DFT-D4 counterparts, with MDs of $0.00 \AA, 0.01 \AA$ and $0.01 \AA$, respectively. Similarly, the MDs for $\omega B 97 X-V$ and its DFT-D3(BJ) and DFT-D4 counterparts are $-0.01 \AA, 0.00 \AA$ and $0.00 \AA . \omega B 97 \mathrm{M}-\mathrm{V}$ also underperforms compared to its DFT-D3(BJ) and DFT-D4 counterparts, with MDs of $-0.02 \AA,-0.01 \AA$ and $-0.01 \AA$. Lastly, we make a comparison with the study of Goerigk and Reimers. ${ }^{52}$ The hybrid functionals they included were PBE0, ${ }^{82183}$ B3LYP, PW6B95 and BHLYP, ${ }^{84}$ with the cc-pVTZ basis set and the DFT-D3(BJ) correction. Their respective MDs are $-0.04,-0.02,0.01$ and $-0.02 \AA$, respectively. We show that B97M-V, $\omega$ B97XD3(BJ) and $\omega$ B97X-D4 all surpass these, with all having deviations of $0.00 \AA$. Table 6 also shows all relevant MADs, which are the absolute values of their respective MDs, which 
demonstrates that the MDs around $0 \AA$ are not a result of spurious error compensation.

\subsubsection{TMC32}

The final geometry benchmark set in our study is the TMC32 set of transition-metal complexes. $\frac{54}{5}$ This set may give us another indication as to how the DFT-D4 methods may differ from their DFT-D3(BJ) counterparts, particularly as this set also contains three openshell complexes. The TMC32 experimental reference data comprise 50 interatomic distances across the 32 complexes. The systems $\mathrm{V}\left(\mathrm{NMe}_{2}\right)_{4}, \mathrm{Cr}\left(\mathrm{C}_{6} \mathrm{H}_{6}\right)_{2}, \mathrm{CoH}(\mathrm{CO})_{4}$ and $\mathrm{Cu}(\text { acac })_{2}$ were not able to be converged with the RI-J approximation for B97M-V, $\omega$ B97X-V and $\omega$ B97MV. Hence, we optimised those geometries without it. All other cases optimised successfully except for $\mathrm{Cu}(\mathrm{acac})_{2}$ for $\omega \mathrm{B} 97 \mathrm{M}-\mathrm{V}$ - this is omitted from our analysis, leaving us with 31 systems and 49 interatomic distances for $\omega \mathrm{B} 97 \mathrm{M}-\mathrm{V}$.

Grimme and co-workers assessed semi-empirical quantum mechanical methods for structures, vibrational frequencies and NCIs for large molecular systems in 2017. 55 Their therein presented GFN-xTB method has an MAD of $0.051 \AA$ for TMC32. In Bühl and Kabrede's original TMC32 study, TPSS ${ }^{85}$ was shown to be most accurate of all methods assessed with a TZ AO basis set, with an MAD of $0.012 \AA$. Figure 3 shows the MADs of our assessed methods. The individual distances and other statistical measures can be found in the SI in Table S23. Herein, we observe the best-performing methods in our study to be B97M-D4 and B97M-V, with MADs of $0.027 \AA$, with B97M-D3(BJ) having an MAD of $0.029 \AA$. We note that PBE-D3(BJ) also has an MAD of $0.029 \AA$. $\omega$ B97X-D3(BJ) and $\omega$ B97X-D4 have MADs of $0.034 \AA$, slightly higher than $\omega$ B97X-V, with $0.033 \AA$. In contrast, $\omega$ B97M-D3(BJ) and $\omega$ B97M-D4 have MADs of $0.030 \AA$, slightly lower than $\omega$ B97M-V, with $0.032 \AA$.

We can conclude that DFT-D4 matches or slightly outperforms DFT-D3(BJ) for geometries of transition-metal complexes with B97M, $\omega$ B97X and $\omega$ B97M. The most viable methods were found to be B97M-D4 and B97M-V, with MADs of $0.027 \AA$, followed by B97M-D3(BJ) and PBE-D3(BJ), with MADs of $0.029 \AA$. 


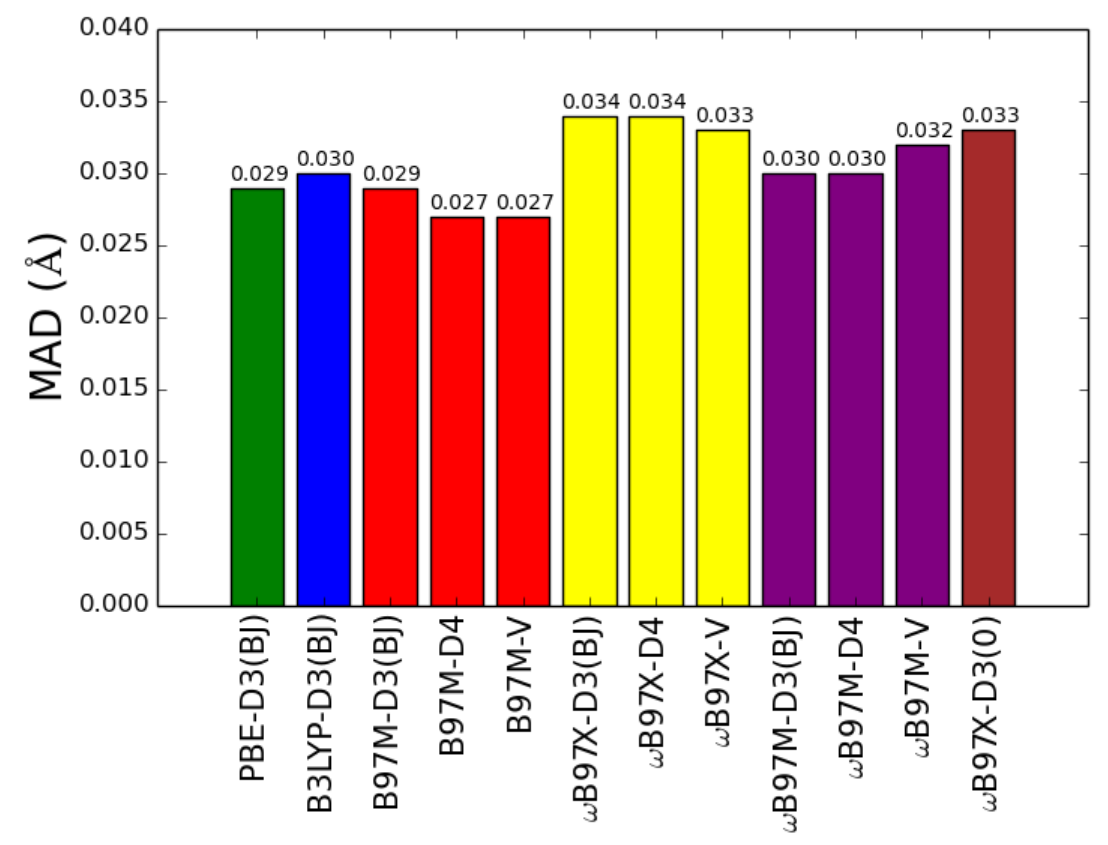

Figure 3: Mean absolute deviations for the various methods tested in this study for the TMC32 benchmark set (kcal/mol). The def2-TZVPD AO basis set was used.

\section{Summary}

We determined damping parameters to build DFT-D4 counterparts of the leading metaGGA functional B97M-V $\mathrm{V}^{36}$ and the leading hybrid functionals $\omega \mathrm{B} 97 \mathrm{X}-\mathrm{V}^{37}$ and $\omega \mathrm{B} 97 \mathrm{M}-$ $\mathrm{V} . \stackrel{38}{38}$ We extensively assessed these DFT-D4 variants together with their DFT-D3(BJ) and original VV10-type counterparts ${ }^{11}$ for energetics and geometries. Our analysis comprised the GMTKN55 database and two additional benchmark sets for energetics, namely for enzymatically catalysed and metal-organic reactions, as well as a four geometry benchmark sets for noncovalently bound dimers and trimers, peptide conformers, water hexamers, and transition-metal complexes.

We found several promising results with the DFT-D4 counterparts for energetics. When comparing our results with the large number of datapoints previously published by us for GMTKN55. $\frac{3|4| 9 \mid 11}{1}$ we found either B97M-D4, $\omega$ B97X-D4 and $\omega$ B97M-D4 to be amongst the top-three assessed DFAs per functional class and category-including the entire database 
itself - a total of 10 times. We can especially point out that B97M-D4 and $\omega$ B97M-D4 are now the most accurate meta-GGA and hybrid functionals assessed for the calculation of barrier heights. B97M-D4 is now the best assessed meta-GGA functional for the intermolecular noncovalent interactions category of GMTKN55. For the entire GMTKN55 database B97M-D4 now ranks in second position amongst meta-GGA functionals. While the DFT-D4 versions of all three functionals do not outperform their DFT-D3(BJ) and "-V" counterparts, they can still be safely used for enzymatically catalysed reactions. Looking at the organometallic REs of MOR41, we see that particularly B97M-D4 is significantly better than B97M-D3(BJ), surpassing B97M-V, while $\omega \mathrm{B} 97 \mathrm{M}-\mathrm{V}$ and $\omega \mathrm{B} 97 \mathrm{X}-\mathrm{V}$ outperform their DFT-D3(BJ) and DFT-D4 counterparts for this set.

Both the DFT-D3(BJ) and DFT-D4 versions of B97M, $\omega$ B97X and $\omega$ B97M were assessed for geometries for the first time. Our analysis suggests that these methods can safely be used for geometries in general. We note that B97M-D3(BJ), B97M-D4 and $\omega$ B97X-D4 surpass previously benchmarked density functionals for the peptide geometries of the P26 benchmark set, and B97M-V, $\omega \mathrm{B} 97 \mathrm{X}-\mathrm{D} 3(\mathrm{BJ})$ and $\omega \mathrm{B} 97 \mathrm{X}-\mathrm{D} 4$ surpass previously benchmarked density functionals for the book, cage and prism water hexamer geometries. The DFT-D4 and DFTD3(BJ) versions match or slightly outperform the original VV10 variants for the TMC32 set for transition-metal complexes.

Overall, B97M-D4 appears to be a very reliable meta-GGA functional for energetics and geometries. Additionally, we remind the reader that the DFT-D3(BJ) and DFT-D4 counterparts of B97M-V, $\omega \mathrm{B} 97 \mathrm{X}-\mathrm{V}$ and $\omega \mathrm{B} 97 \mathrm{M}-\mathrm{V}$ have the benefit of being computationally more efficient than their fully self-consistent VV10-based counterparts. Given their computational efficiency we therefore recommend the safe use of the DFT-D3(BJ) and DFT-D4 versions for geometries. This is achievable with ORCA4.1 and above following the instructions provided in Fig. S1. In the future, we may also include the DFT-D4 variants with their own unique keywords in an upcoming ORCA release. 


\section{Acknowledgements}

We thank Dale Lonsdale and Anwen Xu for some preliminary calculations with the DFTD3(BJ) methods. AN would like to thank The University of Melbourne for a Melbourne Research Scholarship and a Research Training Program Award. LG acknowledges generous allocation of resources by the National Computational Infrastructure (NCI) National Facility within the National Computational Merit Allocation Scheme (project ID: fk5). This research was also supported by the sustaining and strengthening merit-based access to the NCI LIEF Grant (LE190100021) facilitated by The University of Melbourne.

\section{References}

(1) Kohn, W.; Sham, L. J. Phys. Rev. 1965, 140, A1133-A1138.

(2) Becke, A. D. J. Chem. Phys. 2014, 140, 18A301.

(3) Goerigk, L.; Hansen, A.; Bauer, C.; Ehrlich, S.; Najibi, A.; Grimme, S. Phys. Chem. Chem. Phys. 2017, 19, 32184-32215.

(4) Goerigk, L.; Mehta, N. Aust. J. Chem. 2019, 72, 563-573.

(5) Goerigk, L.; Grimme, S. Phys. Chem. Chem. Phys. 2011, 13, 6670-6688.

(6) Haoyu, S. Y.; He, X.; Li, S. L.; Truhlar, D. G. Chem. Sci. 2016, 7, 5032-5051.

(7) Peverati, R.; Truhlar, D. G. Philos. Trans. R. Soc., A 2014, 372, 20120476.

(8) Mardirossian, N.; Head-Gordon, M. Mol. Phys. 2017, 115, 2315-2372.

(9) Mehta, N.; Casanova-Páez, M.; Goerigk, L. Phys. Chem. Chem. Phys. 2018, 20, 2317523194.

(10) Mardirossian, N.; Head-Gordon, M. J. Chem. Phys. 2018, 148, 241736. 
(11) Najibi, A.; Goerigk, L. J. Chem. Theory Comput. 2018, 14, 5725-5738.

(12) Chan, B. J. Chem. Theory Comput. 2018, 14, 4254-4262.

(13) Gould, T. Phys. Chem. Chem. Phys. 2018, 20, 27735-27739.

(14) Morgante, P.; Peverati, R. J. Comput. Chem. 2019, 40, 839-848.

(15) Morgante, P.; Peverati, R. Phys. Chem. Chem. Phys. 2019, 21, 19092-19103.

(16) Santra, G.; Sylvetsky, N.; Martin, J. M. L. J. Phys. Chem. A. 2019, 123, 5129-5143.

(17) Martin, J. M. L.; Santra, G. Isr. J. Chem. 2019, published online, DOI: 10.1002/ijch.201900114.

(18) Kristyán, S.; Pulay, P. Chem. Phys. Lett. 1994, 229, 175 - 180.

(19) Pérez-Jordá, J.; Becke, A. D. Chem. Phys. Lett. 1995, 233, 134 - 137.

(20) Hobza, P.; S̆poner, J.; Reschel, T. J. Comput. Chem. 1995, 16, 1315-1325.

(21) Grimme, S.; Hansen, A.; Brandenburg, J. G.; Bannwarth, C. Chem. Rev. 2016, 116, 5105-5154.

(22) Ángyán, J.; Dobson, J.; Jansen, G.; Gould, T. London Dispersion Forces in Molecules, Solids and Nano-structures; Theoretical and Computational Chemistry Series; The Royal Society of Chemistry, 2020.

(23) Grimme, S.; Antony, J.; Ehrlich, S.; Krieg, H. J. Chem. Phys. 2010, 132, 154104.

(24) Grimme, S.; Ehrlich, S.; Goerigk, L. J. Comput. Chem. 2011, 32, 1456-1465.

(25) Goerigk, L. In Non-Covalent Interactions in Quantum Chemistry and Physics; Otero de la Roza, A., DiLabio, G. A., Eds.; Elsevier: Amsterdam, 2017; pp 195 - 219.

(26) Caldeweyher, E.; Bannwarth, C.; Grimme, S. J. Chem. Phys. 2017, 147, 034112. 
(27) Caldeweyher, E.; Ehlert1, S.; Hansen, A.; Neugebauer, H.; Spicher, S.; Bannwarth, C.; Grimme, S. J. Chem. Phys. 2019, 150, 154112.

(28) Dion, M.; Rydberg, H.; Schröder, E.; Langreth, D. C.; Lundqvist, B. I. Phys. Rev. Lett. 2004, 92, 246401.

(29) Dion, M.; Rydberg, H.; Schröder, E.; Langreth, D. C.; Lundqvist, B. I. Phys. Rev. Lett. 2005, 95, 109902.

(30) Vydrov, O. A.; Van Voorhis, T. J. Chem. Phys. 2010, 133, 244103.

(31) Hujo, W.; Grimme, S. J. Chem. Theory Comput. 2011, 7, 3866-3871.

(32) Hujo, W.; Grimme, S. Phys. Chem. Chem. Phys. 2011, 13, 13942-13950.

(33) Goerigk, L. J. Chem. Theory Comput 2014, 10, 968-980.

(34) Risthaus, T.; Grimme, S. J. Chem. Theory Comput. 2013, 9, 1580-1591.

(35) Mehta, N.; Abrahams, B. F.; Goerigk, L. Chem. - Asian J. 2020, 15, 1301-1314.

(36) Mardirossian, N.; Head-Gordon, M. J. Chem. Phys. 2015, 142, 074111.

(37) Mardirossian, N.; Head-Gordon, M. Phys. Chem. Chem. Phys. 2014, 16, 9904-9924.

(38) Mardirossian, N.; Head-Gordon, M. J. Chem. Phys. 2016, 144, 214110.

(39) Becke, A. D. J. Chem. Phys. 1997, 10\%, 8554-8560.

(40) Wappett, D. A.; Goerigk, L. J Phys. Chem. A 2019, 123, 7057-7074.

(41) Iron, M. A.; Janes, T. J. Phys. Chem. A 2019, 123, 3761-3781.

(42) Kraus, P.; Obenchain, D. A.; Frank, I. J. Phys. Chem. A 2018, 122, 4894-4901.

(43) Jurecka, P.; Sponer, J.; Cerny, J.; Hobza, P. Phys. Chem. Chem. Phys. 2006, 8, 19851993. 
(44) С̆ezác̆, J.; Riley, K. E.; Hobza, P. J. Chem. Theory Comput. 2011, 7, 2427-2438.

(45) Hujo, W.; Grimme, S. J. Chem. Theory Comput. 2013, 9, 308-315.

(46) Neese, F. Wiley Interdiscip. Rev.: Comput. Mol. Sci. 2012, 2, 73-78.

(47) Neese, F. Wiley Interdiscip. Rev.: Comput. Mol. Sci. 2018, 8, e1327.

(48) Wappett, D. A.; Goerigk, L. J Phys. Chem. A 2020, 124, 1062.

(49) Dohm, S.; Hansen, A.; Steinmetz, M.; Grimme, S.; Checinski, M. P. J. Chem. Theory Comput. 2018, 14, 2596-2608.

(50) Kraus, P.; Obenchain, D. A.; Frank, I. J. Phys. Chem. A 2018, 122, 1077-1087.

(51) Valdes, H.; Pluhackova, K.; Pitonak, M.; Rezac, J.; Hobza, P. Phys. Chem. Chem. Phys. 2008, 10, 2747-2757.

(52) Goerigk, L.; Reimers, J. R. J. Chem. Theory Comput. 2013, 9, 3240-3251.

(53) Perez, C.; Muckle, M. T.; Zaleski, D. P.; Seifert, N. A.; Temelso, B.; Shields, G. C.; Kisiel, Z.; Pate, B. H. Science 2012, 336, 897-901.

(54) Bühl, M.; Kabrede, H. J Chem. Theory Comput. 2006, 2, 1282-1290.

(55) Grimme, S.; Bannwarth, C.; Shushkov, P. J Chem. Theory Comput. 2017, 13, 19892009 .

(56) Perdew, J. P.; Burke, K.; Ernzerhof, Phys. Rev. Lett. 1996, 77, 3865-3868.

(57) Becke, A. D. J. Chem. Phys. 1993, 98, 5648-5652.

(58) Stephens, P. J.; Devlin, F. J.; Chabalowski, C. F.; Frisch, M. J. J. Phys. Chem. 1994, 98, 11623-11627.

(59) Lin, Y. S.; Li, G. D.; Mao, S. P.; Chai, J. D. J. Chem. Theory Comput. 2013, 9, 263-272. 
(60) Gráfová, L.; Piton̆ák, M.; Rezác̆, J.; Hobza, P. J. Chem. Theory Comput. 2010, 6, $2365-2376$.

(61) Taylor, D. E.; Ángyán, J. G.; Galli, G.; Zhang, C.; Gygi, F.; Hirao, K.; Song, J. W.; Rahul, K.; Anatole von Lilienfeld, O.; Podeszwa, R. et al. J. Chem. Phys. 2016, 145, 124105.

(62) DFT-D4 Version 2.0, E. Caldeweyher, S. Ehlert and S. Grimme, Mulliken Center for Theoretical Chemistry, University of Bonn (Germany), 2019.

(63) Weigend, F.; Ahlrichs, R. Phys. Chem. Chem. Phys. 2005, 7, 3297-3305.

(64) Ghasemi, S. A.; Hofstetter, A.; Saha, S.; Goedecker, S. Phys. Rev. B 2015, 92, 045131.

(65) Kendall, R. A.; Dunning, T. H.; Harrison, R. J. J. Chem. Phys. 1992, 96, 6798-6806.

(66) Rappoport, D.; Furche, F. J. Chem. Phys. 2010, 133, 134105.

(67) Dunning, T. H. J. Chem. Phys. 1989, 90, 1007-1023.

(68) Shao, Y.; Gan, Z.; Epifanovsky, E.; Gilbert, A. T. B.; Wormit, M.; Kussmann, J.; Lange, A. W.; Behn, A.; Deng, J.; Feng, X. et al. Mol. Phys. 2015, 113, 184-215.

(69) Eichkorn, K.; Treutler, O.; Öhm, H.; Häser, M.; Ahlrichs, R. Chem. Phys. Lett. 1995, 240, 283-290.

(70) Izsak, R.; Neese, F. J. Chem. Phys. 2011, 135, 144105.

(71) Perdew, J. P.; Schmidt, K. AIP Conference Proceedings 2001, 577, 1-20.

(72) Brandenburg, J. G.; Bannwarth, C.; Hansen, A.; Grimme, S. J. Chem. Phys. 2018, 148,064104 .

(73) Kromann, J. C.; Christensen, A. S.; Cui, Q.; Jensen, J. H. PeerJ. 2016, 4, e1994.

(74) Liao, R. Z.; Yu, J. G.; Himo, F. J. Chem. Theory Comput. 2011, 7, 1494-1501. 
(75) Georgieva, P.; Himo, F. J. Comput. Chem. 2010, 31, 1707-1714.

(76) Sevastik, R.; Himo, F. Bioorg. Chem. 2007, 35, 444-457.

(77) Chen, S. L.; Fang, W. H.; Himo, F. J. Phys. Chem. B 2007, 111, 1253-1255.

(78) Hopmann, K. H.; Himo, F. J. Chem. Theory Comput. 2008, 4, 1129-1137.

(79) Riplinger, C.; Sandhoefer, B.; Hansen, A.; Neese, F. J. Chem. Phys. 2013, 139, 134101.

(80) Goerigk, L.; Collyer, C. A.; Reimers, J. R. J. Phys. Chem. B 2014, 118, 14612-14626.

(81) Zhao, Y.; Truhlar, D. G. J. Phys. Chem. A 2005, 109, 5656-5607.

(82) Adamo, C.; Barone, V. J. Chem. Phys. 1999, 110, 6158-6170.

(83) Ernzerhof, M.; Scuseria, G. E. J. Chem. Phys. 1999, 110, 5029-5036.

(84) Becke, A. D. J. Chem. Phys. 1993, 98, 1372-1377.

(85) Tao, J.; Perdew, J. P.; Staroverov, V. N.; Scuseria, G. E. Phys. Rev. Lett. 2003, 91, 146401. 